\title{
CLINICO-MYCOLOGICAL STUDY OF DERMATOPHYTIC SKIN INFECTIONS IN A TERTIARY CARE CENTRE
}

\author{
Shanimole Puthenpurayil Ebrahimkutty ${ }^{1}$, Shoba Kurian Pulikkottil2, Dani Kochuplapparambil Thampi ${ }^{3}$
}

${ }_{1}^{1}$ Assistant Professor, Department of Microbiology, Government Medical College, Kottayam, Kerala.

2 Professor, Department of Microbiology, Government Medical College, Kottayam, Kerala.

3 Senior Resident, Department of Microbiology, Government Medical College, Kottayam, Kerala.

\begin{tabular}{l}
\hline ABSTRACT \\
\hline BACKGROUND \\
Skin infections are the most common infections occurring in developing countries, of which dermatophytosis are of particular \\
concern in tropics. The prevalence of dermatophytic infections varies with different geographic areas and climatic conditions. \\
Hence, identification of aetiological agents is important for diagnosis and treatment as well as for studying epidemiological \\
characteristics in a region. \\
Objectives- \\
1. To isolate and identify the dermatophytes infecting skin. \\
2. To compare two different culture media, namely Sabouraud's Dextrose Agar (SDA), Hi-Media Laboratories, Mumbai) with \\
Actidione and Dermatophyte Test Medium (DTM, Hi-Media Laboratories).
\end{tabular}

\section{MATERIALS AND METHODS}

This is a cross-sectional study conducted in patients attending the Outpatient Department of Dermatology and Venereology, Government Medical College, Kottayam, Kerala, India, with clinical features of dermatophytic skin infections from January 2009 December 2009. Skin scrapings were subjected to direct microscopy by $10 \%$ potassium hydroxide (KOH) and cultured on SDA with Actidione (Hi-Media Laboratories) and DTM (Hi-Media Laboratories).

\section{RESULTS}

The total number of samples in this study was 115 , of which $76(66.1 \%)$ samples were positive by direct microscopy and 50 (43.5\%) were positive by culture. The most common clinical type was tinea corporis 46 (40\%). Out of the 50 isolates, Trichophyton rubrum 24 (48\%) was the most common species followed by Trichophyton mentagrophytes 20 (40\%), Epidermophyton floccosum 4 (8\%), Trichophyton violaceum 1 (2\%) and Microsporum gypseum 1 (2\%). Nearly, 85.4\% of the dermatophytes were isolated on DTM within 5 - 10 days of incubation, whereas 45.7\% were isolated on SDA with Actidione within 5 - 10 days of incubation.

\section{CONCLUSION}

DTM can be used as a rapid screening medium for the isolation and identification of dermatophytes compared to SDA with Actidione. But DTM is inferior to SDA with Actidione for species level identification of dermatophytes.

\section{KEYWORDS}

Dermatophytosis, Trichophyton, Microsporum.

HOW TO CITE THIS ARTICLE: Ebrahimkutty SP, Pulikkottil SK, Thampi DK. Clinico-mycological study of dermatophytic skin infections in a tertiary care centre. J. Evolution Med. Dent. Sci. 2018;7(08):1008-1011, DOI: 10.14260/jemds/2018/230

\section{BACKGROUND}

Dermatophytosis is by far the most common superficial infection affecting keratinised tissue of stratum corneum involving hair, nail and skin. Dermatophytes are filamentous fungi, which are physiologically adapted for growth in keratin causing dermatophytosis commonly known as "Tinea" and "Ringworm" infection.[1] Though dermatophytosis is worldwide in distribution, it is more prevalent in tropical and subtropical countries.[2,3] Environmental conditions and living style of people play a role in the disease. Fungal infection of

'Financial or Other Competing Interest': None.

Submission 11-01-2018, Peer Review 02-02-2018,

Acceptance 10-02-2018, Published 19-02-2018.

Corresponding Author:

Shanimole Puthenpurayil Ebrahimkutty,

Assistant Professor,

Department of Microbiology,

Government Medical College,

Kottayam, Kerala.

E-mail: shanisdr@gmail.com

DOI: $10.14260 /$ jemds $/ 2018 / 230$ skin and its appendages are more prevalent in India due to favourable climatic conditions like temperature and humidity. In India which is a tropical country, the cause of dermatophytosis is adversely influenced by economic factors like poverty, poor hygiene and social conditions like overcrowding. Nature of dermatophytosis may change with passage of time, living population, evolution of preventive measures and hygienic conditions in society. ${ }^{[4]}$ Dermatophytes are septate moulds with three anamorphic genera- Trichophyton, Microsporum and Epidermophyton. Depending on its origin it is divided into geophilic, zoophilic and anthropophilic species.[5] Each genus has a distinctive pattern of conidiation that is considered characteristic. Differentiation relies heavily on the kind of microconidia and macroconidia produced, the size, shape, septation and attachment to the hyphae of the conidia and the vegetative hyphal structures present. Microsporum can produce both macroconidia and microconidia. Typical macroconidia are fusiform or spindle shaped. The wall of macroconidia is thick and echinulate with upto 15 septa. Microconidia are less commonly seen. They are pyriform or clavate and are born directly on the hyphae or on short conidiophores. 
Trichophyton also produce both macroconidia and microconidia. Microconidia, usually more abundant than macroconidia may be globose, pyriform or clavate and are borne singly along the sides of the hyphae or in grape-like clusters. Macroconidia, when present have smooth, thin walls and may be elongate and pencil shaped, clavate, fusiform or cylindrical with 2 to 12 septa. The genus Epidermophyton never produces microconidia. The macroconidia have thin, smooth walls and contain one to five septa resembling heaver's tails or snowshoes.[6] A single species may be involved in several disease types, each with its distinctive pathology. Dermatophytosis causes inflammatory response and intense itching lesions of cosmetic importance. The severity of the disease depends on the strain or the species of the dermatophyte and the susceptibility of the host to the fungus. Immunocompromised states due to underlying malignancy, administration of steroids or immunosuppressive drugs, acquired immunodeficiency syndrome or endocrinological disorders such as diabetes mellitus and Cushing's disease can lead to generalised and atypical presentations that can be confused with other skin disorders. ${ }^{[4]}$ Although, not life-threatening, its severity can cause great discomfort. A correct diagnosis of the aetiological agents of dermatophytosis is therefore important to initiate treatment and is also essential for epidemiological purposes. As not much work has been done in recent past in this field and the incidence and species prevalence vary from place to place and dermatophytic skin infections are more frequent when compared to those of hair and nails, the present study was aimed to identify the clinico-mycological pattern of dermatophytic skin infections.

\section{Objectives}

1. To isolate and identify the dermatophytes infecting skin.

2. To compare two different culture media, namely Sabouraud's Dextrose Agar (SDA, Hi-Media Laboratories, Mumbai) with Actidione and Dermatophyte Test Medium (DTM, Hi-Media Laboratories).

\section{MATERIALS AND METHODS}

A total of 115 clinically suspected cases of dermatophytic skin infections who attended the Dermatology and Venereology Outpatient Department of Government Medical College, Kottayam, Kerala for a period of one year from January 2009 December 2009 were included in the study. A detailed history of the patients including age, sex, income, occupation, duration of disease, history of recurrence, habits, associated diseases, family history and history of pets was taken. Patients with history of use of antifungals, both topical and systemic for 3 weeks or more were excluded from the study. Ethical clearance was obtained.

\section{Study Design}

Cross-sectional study.

Skin scrapings from the active edge of the lesions were collected. One portion of the sample was first subjected to direct microscopy by $10 \%$ potassium hydroxide $(\mathrm{KOH})$ wet mount. The rest of the samples were cultured on- a) Sabouraud's Dextrose Agar (SDA) with chloramphenicol and Actidione (Hi-Media Laboratories), b) Dermatophyte test medium (DTM; Hi-Media Laboratories). Samples were inoculated into two sets of these culture media. One set was incubated at $37^{\circ} \mathrm{C}$ and another at $25^{\circ} \mathrm{C}$ for four weeks. After initial inoculation and incubation, cultures were examined every 2 - 3 days during the first week and weekly thereafter. The colony was identified by looking the texture, surface colour of the colony, colour of the reverse of the colony and presence of any pigment that diffuses into the medium. A tease mount was made as soon as sufficient growth was evident on the medium. i.e. Place a drop of lactophenol cotton blue (LPCB) on a clean glass slide. With a sterile bent dissecting needle, remove a small portion of the colony from the agar surface and place it in the drop of LPCB. With two dissecting needles, gently tease apart the mycelial mass of colony on the slide, cover with coverslip and observe first under low power, then under high power objective. Dermatophytes were identified by the characteristic conidiation, presence or absence of microconidia and macroconidia, shape, size, septation, attachment to the hyphae and characteristic hyphal structures. If the morphology cannot be identified by tease mount technique, slide culture technique was performed. For slide culture technique, an agar-block is placed on a glass slide and inoculated, then covered with a coverslip. Then it is incubated in a glass petri dish and water is added to provide necessary moisture. Additional tests like urea hydrolysis and in-vitro hair perforation tests were done if necessary. ${ }^{66,7]}$

\section{Urea Hydrolysis Test}

Ability to hydrolyse urea provides an additional aid in differentiation of Trichophyton mentagrophyte (Urease positive) from Trichophyton rubrum (Urease negative). Here, the fungal colony is inoculated in Christensen's urea agar and look for development of pink colour. Urease positive organisms produce an alkaline reaction indicated by pink colour.

\section{In-Vitro Hair Perforation Test}

Ability of a fungus to perforate natural hair helps to differentiate between T. mentagrophyte from T. rubrum. Here, place $1 \mathrm{~cm}$ long fragments of healthy human hair, preferably from a child less than 12 years old in a petri dish and sterilise by autoclaving at $15 \mathrm{lb} / \mathrm{in}^{2}$ for 15 minutes. Place $8-10$ of the sterile hair fragments in a sterile $50 \mathrm{~mL}$ screw cap tube. Add $20-25 \mathrm{~mL}$ of sterile distilled water and $0.1 \mathrm{~mL}$ of filter sterilised yeast extract. Place several fragments of the fungal culture in the tube. Incubate at room temperature for 4 weeks. Examine weekly intervals by placing one or two hairs from the culture onto a slide with a drop of LPCB and a coverslip. Look for wedge-shaped perforations caused by hyphae that penetrates the hairs perpendicularly. The hair is perforated by T. mentagrophytes, but not by T. rubrum. ${ }^{[7]}$

\section{Dermatophyte Test Medium (DTM)}

It is a partially selective medium for dermatophytes and contain antibiotics to inhibit bacteria and cycloheximide to inhibit many saprophytic fungi. Phenol red is used as an indicator. Dermatophytes produce alkaline metabolic products. The indicator detects these and the medium changes from yellow-to-reddish orange or red.[6,7] 


\section{RESULTS}

Among 115 patients, 59 (51.3\%) were males and 56 (48.7\%) were females. The peak incidence of dermatophytic skin infections was seen in the age group of $30-49$ yrs. (38.2\%). In this study, maximum number of patients were active workers 60 (52.2\%) followed by housewives 31 (26.9\%).

The most common clinical presentation was tinea corporis 46 (40\%) followed by mixed infections 35 (30.4\%). Among the mixed infections, the most common pattern was a combination of tinea corporis and tinea cruris 20 (17.3\%) [Table 1].

Direct smear positivity (KOH) was found in 76 (66.1\%), whereas culture positivity was found in 50 (43.5\%). Trichophyton rubrum was the most common isolate, 24 (48\%) followed by Trichophyton mentagrophytes 20 (41.6\%). Other isolates are Epidermophyton floccosum 4 (8\%), Trichophyton violaceum 1 (2\%) and Microsporum gypseum 1 (2\%) [Table 2]. Out of 20 culture positive cases of tinea corporis, T. rubrum was the most common isolate 10 (50\%). Out of 15 culture positive cases of mixed infections, T. rubrum was the most common isolate $8(53.3 \%)$.

In this study, 48 (96\%) dermatophytes were isolated from DTM (Hi-Media Laboratories) and 46 (92\%) dermatophytes were isolated from SDA with Actidione [Table-3]. Two isolates of T. mentagrophyte did not grow on DTM. Three isolates of T. mentagrophytes and one isolate of $T$. rubrum did not grow on SDA with Actidione (Hi-Media Laboratories) [Table 4].

In the present study, it was observed that $41(85.4 \%)$ dermatophytes were isolated from DTM within 5 - 10 days of incubation, whereas 21 (45.7\%) dermatophytes were isolated from SDA with Actidione during the same period [Table 5]. It was also observed that conidiation was poor in DTM compared to SDA with actidione. Therefore, dermatophyte grown on DTM has to be subcultured onto SDA with actidione for species identification. It was also observed that when dermatophyte was grown on DTM, the colour of the medium is changed from yellow to red. Therefore, pigment production by dermatophyte like T. rubrum could not be appreciated.

\begin{tabular}{|c|c|c|c|}
\hline SI. No. & Clinical Types & Number & $\mathbf{\%}$ \\
\hline 1 & Tinea corporis & 46 & 40.0 \\
\hline 2 & Mixed pattern & 35 & 30.4 \\
\hline 3 & Tinea cruris & 18 & 15.8 \\
\hline 4 & Tinea manuum & 7 & 6.1 \\
\hline 5 & Tinea faciei & 5 & 4.3 \\
\hline 6 & Tinea barbae & 2 & 1.7 \\
\hline 7 & Tinea pedis & 2 & 1.7 \\
\hline \multicolumn{3}{|c|}{ Table 1. Distribution of Cases according to Clinical } \\
Types \\
\hline
\end{tabular}

\begin{tabular}{|c|c|c|c|}
\hline $\begin{array}{l}\text { SI. } \\
\text { No. }\end{array}$ & Species & Number & $\begin{array}{c}\text { Percentage } \\
(\%)\end{array}$ \\
\hline 1 & Trichophyton rubrum & 24 & 48 \\
\hline 2 & $\begin{array}{l}\text { Trichophyton } \\
\text { mentagrophytes }\end{array}$ & 20 & 40 \\
\hline 3 & Epidermophyton floccosum & 4 & 8 \\
\hline 4 & Trichophyton violaceum & 1 & 2 \\
\hline 5 & Microsporum gypseum & 1 & 2 \\
\hline & Total & 50 & 100 \\
\hline
\end{tabular}


dermatophytes, natural pathogens of humans are the most common cause of human dermatophytosis. The most common of these organisms in most part of the world is $T$. rubrum, which causes tinea corporis particularly in the tropics. This organism that infect glabrous skin spread largely through contact with infected skin scales. Classically, this occurs in bathing areas or shower rooms where large number of individuals share common facilities.[10] This can be a reason for T. rubrum becoming the commonest aetiological agent in this study where overcrowding, tropical climate and humidity are contributory factors. The other less common isolates were Epidermophyton floccosum, Trichophyton violaceum and Microsporum gypseum. Majeed et al[16] and Patel et al[11] reported similar findings in their study in 2016 and 2012 respectively.

In the present study, $96 \%$ of culture positives were grown on DTM and $92 \%$ of culture positives were grown on SDA with Actidione. This was in correlation with the study of Singh and Beena[17] who reported that $98.3 \%$ of culture positives were grown on DTM and $96.55 \%$ of culture positives were grown on SDA. SDA with Actidione is a primary isolation media for fungi, whereas Dermatophyte test medium is a partially selective medium for dermatophytes.[7] In the present study, even though the isolation rate was good in both DTM and SDA with Actidione, the appearance of growth was earlier on DTM. When comparing the rate of growth on SDA with actidione and on DTM, it was observed that $85.4 \%$ dermatophytes were grown on DTM within 5 - 10 days of incubation, whereas only $45.75 \%$ were grown in SDA with Actidione during the same period. Majeed et al reported in 2016 in their study that $86.1 \%$ dermatophytes were grown on DTM within 5 - 10 days of incubation and only $47.05 \%$ were grown in SDA with actidione during the same period.[16] It is also observed in the present study that characteristic conidiation is poor in DTM compared to SDA with actidione. Since conidia formation is better in nutritionally deficient media, fungal growth from DTM has to be subcultured onto SDA with actidione.[6] Therefore, DTM can be used as a screening medium for early identification of dermatophytes. But for species level identification of dermatophytes, SDA with Actidione is preferable compared to DTM. Moreover, red pigment produced by dermatophyte like $T$. rubrum could not be appreciated on DTM as the change produced by growth of any dermatophyte on DTM is change in colour of medium from yellow to red.

\section{Limitation of this Study}

Molecular diagnosis of isolate could not be done. Antifungal susceptibility cannot be done.

\section{CONCLUSION}

The present study highlighted that tinea corporis is the commonest clinical type. Trichophyton rubrum and Trichophyton mentagrophytes are the most common aetiological agents. DTM is a good screening medium in the laboratory diagnosis of dermatophytic infections compared to SDA with Actidione. However, DTM is not a preferable medium for species identification of dermatophytes.

\section{REFERENCES}

[1] Rippon JW. Medical mycology. The pathogenic fungi and pathogenic actinomycetes. $3^{\text {rd }}$ edn. Philadelphia: WB Saunders, 1988:169-275.

[2] Sobbanadri S, Rao DT, Babu KS. Clinical and mycological study of superficial infections at government general hospital, Gauntur and their response to treatment with Hamycin, Dermostatin and Dermamycin. Indian Journal of Dermatology, Venerology and Leprology 1970;36(6):209-14.

[3] Verma KC, Singh K. Dermatomycoses in Rohtak (an analysis of cases). Indian J Dermatol Veneorol 1972;38(6):238-42.

[4] Kumar S, Mallya PS, Kumari P. Clinico mycological study of dermatophytosis in a tertiary care hospital. International Journal of Scientific Study 2014;1(6):2731.

[5] Chandar J. Text book of medical mycology. Dermatophytosis. $3^{\text {rd }}$ edn. City of Publishing New Delhi: Mehta Publishers, 2009:122-46.

[6] Fisher F, Cook NB. Fundamentals of medical mycology. Dermatophytes. WB Saunders, 1998:118-56.

[7] Larone DH. Medically important fungi a guide to identification. Laboratory procedures. $5^{\text {th }}$ edn. Washington DC, ASM press, 2011:339-73.

[8] Sharma R, Jasuja ND, Sharma S. Clinical and mycological study of dermatophytosis in Jaipur. Intl Pharm Sci 2012;4:15-7.

[9] Poluri LV, Indugula JP, Kondapaneni SL. Clinicomycological study of dermatophytosis in South India. J Lab Physicians 2015;7(2):84-9.

[10] Mandell RJ. Douglas and Bennett's principle and practice of infectious diseases. Dermatophytosis and other superficial mycoses. 7 th edn. Philadelphia: Churchill Livingstone Elsevier, 2010;2:3346-8.

[11] Patel P, Mulla S, Patel D, et al. A study of superficial mycoses in Gujarat region. Indian J Med Microbiol 2012;1:85-8.

[12] Bindu V, Pavithran K. Clinico-mycological study of dermatophytosis in Calicut. Indian J Dermatol Venerol Leprol 2002;68(5):259-61.

[13] Kucheria M, Gupta SK, Chhina DK, et al. Clinicomycological profile of dermatophytic infections at a tertiary care hospital in north India. International Journal of Community Health and Medical Research 2016;2(2):17-22.

[14] Gupta CM, Tripathi K, Tiwari S, et al. Current trends of Clinicomycological profile of dermatophytosis in Central India. J Dent Med Sci 2014;13(10):23-6.

[15] Lakshmanan A, Ganeshkumar P, Mohan SR, et al. Epidemiological and clinical pattern of dermatomycoses in rural India. Indian J Med Microbiol 2015;33:134-6.

[16] Majeed N, Narayanankutty S, Rajan R, et al. Clinicomycological study of dermatophytosis in a tertiary care centre. Journal of Academy of Clinical Microbiologists 2016;18(2):110-3.

[17] Singh S, Beena PM. Comparative study of different microscopic techniques and culture media for the isolation of dermatophytes. Indian J Med Microbiol 2003;21(1):21-4. 